\title{
On the isomorphism types of Moebius-Kantor complexes
}

\author{
Sylvain Barré and Mikaël Pichot
}

\begin{abstract}
We study the isomorphism types of simply connected complexes with Moebius-Kantor links using a local invariant called the parity. We show that the parity can be computed explicitly in certain constructions arising from surgery.
\end{abstract}

\section{Introduction}

We call Moebius-Kantor complex a 2-complex with triangle faces, whose links are isomorphic to the Moebius-Kantor graph (Fig. 1).

In this paper we continue our investigations of these complexes, which was begun in [5]. We study the isomorphism type of simply connected MoebiusKantor complexes, and aim to prove that

(a) there are infinitely many isomorphism types with a cocompact automorphism group

(b) there are uncountably many isomorphism types, in general.

These statements should be compared to similar ones, for Euclidean Tits buildings, especially in the rank 2 case.

The second statement (b) is reminiscent of the "free constructions" of Ronan [9] for buildings of type $\tilde{A}_{2}$ (and more generally for buildings lacking irreducible spherical residues of type $A_{3}, C_{3}$ or $H_{3}$ ), which can be used in particular to show the existence of uncountably many such buildings whose residue have a fixed finite order $q$. The main tool to establish free constructions is a prescription theorem of a local invariant of the complex. For buildings of type $\tilde{A}_{2}$, Ronan has proved such a theorem in [9], showing that one can prescribe the residues at vertices arbitrarily, which can furthermore be provided by any set of projective planes of fixed order $q$.

Furthermore, in the case $q=2$, Tits has shown in [14] that there are precisely two isomorphism types of spheres of radius 2 , and the type of spheres of radius 2 can also be used as a local invariant by $[1,2]$. 


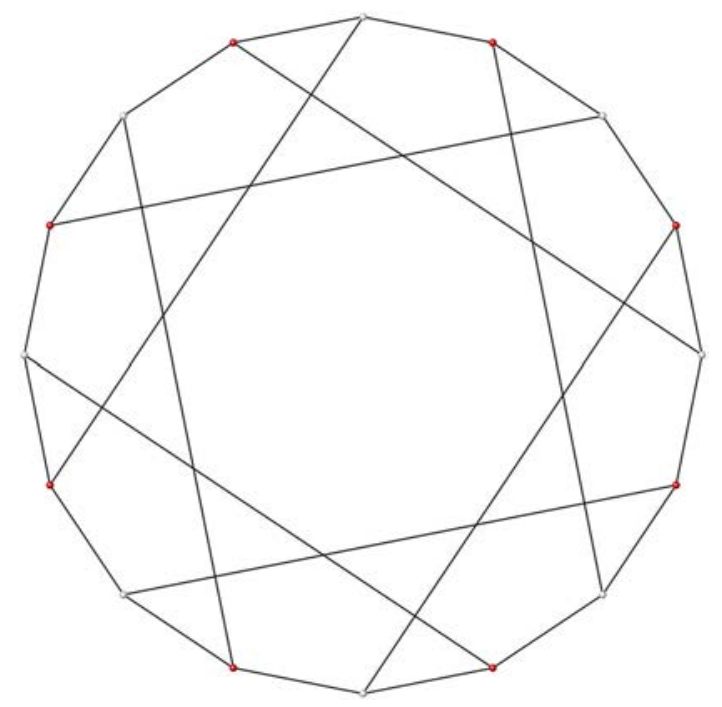

Figure 1 The Moebius-Kantor graph

The use of spheres of radius 2 is not very practical in our situation. Our results will in fact show that there are at least 174,163 isomorphism types of such spheres. We shall rely instead on a local invariant which makes use of the intermediate rank properties of Moebius-Kantor complexes, and in particular, how the roots of rank 2 (and those of rank $\neq 2$ ) are organized in the complex. The invariant is defined in Sect. 2 and called the parity. A free prescription theorem for the parity is established in Sect. 5 .

The first statement (a) is also true for Euclidean buildings. A way to prove it, which is in fact the only way we know of, even in the rank 2 case, is to use Euclidean buildings associated with non isomorphic local fields. For example, if $\left(K_{r}\right)_{r}$ is a sequence of totally ramified finite extensions of $\mathbb{Q}_{p}$ which are pairwise nonisomorphic, then (by the classical results of Tits [11]) the Euclidean Tits buildings $X_{r}$ of $\mathrm{PSL}_{d}\left(K_{r}\right)$ will be also pairwise nonisomorphic.

For Moebius-Kantor complexes, constructions using local fields are not available. However, an alternative approach for building such complexes was proposed in [7], using surgery theory. This construction provides compact Moebius-Kantor complexes, and therefore simply connected ones with cocompact automorphism groups, taking universal covers. The question addressed in (a), which was raised in a recent talk by one of us, is to distinguish these complexes up to isomorphism. The problem, of course, is that different constructions may lead to isomorphic universal covers. In Sect. 4, we show that the parity can be computed explicitly in these constructions, and that it provides a way to distinguish these complexes up to isomorphism.

Note that infinitely many of the Euclidean buildings $X_{r}$ defined in the previous paragraph coincide on arbitrary large balls, since there are only finitely many 
isomorphism types of balls of radius $n$ in this case. In order to prove (a), we must in fact show that a similar construction, where the complexes coincide on arbitrarily large balls, can be done in the case of Moebius-Kantor complexes (with cocompact automorphism group). This is because the existence of infinitely many isomorphism types of Moebius-Kantor complexes with cocompact automorphism group, as stated in (a), implies the same statement where furthermore, the complexes coincide on arbitrary large finite balls. (Another way to phrase this is to use the space of simply connected Moebius-Kantor complexes, an analog to the space of triangle buildings $E_{q}$ of $[3,4]$, which is a compact space.) Therefore, we might as well look directly for such complexes, which is what we shall do.

We note however that, for Moebius-Kantor complexes, the quotient by the automorphism group in (a) has to be arbitrary large, because the automorphism group is uniformly discrete. An analogous statement for buildings of type $\tilde{A}_{2}$ is not known to hold: questions regarding the existence or number of buildings, whose quotient by their automorphism group has a prescribed, e.g., arbitrarily large, number of vertices, are generally open. This is one of our motivations to look for new constructions techniques.

The following is a further analogy between the rank $\frac{7}{4}$ constructions and the local field constructions for $X_{r}$. The buildings $X_{r}$ can be distinguished up to isomorphism, because the field $K_{r}$ can be recovered abstractly, by the results of Tits [11], from the building at infinity. However, it is not clear a priori how distant from each other these complexes are in the space of buildings (say in the $\tilde{A}_{2}$ case). A similar phenomenon is also occurs in our rank $\frac{7}{4}$ constructions. It is not clear a priori how distant from each other the complexes we build will be in the space of Moebius-Kantor complexes, since there might a priori exist "exotic" isomorphisms between the spheres of large radius which we have not detected. Thus, we only prove an injectivity result, see the proof of Theorem 4.1, which explains that the final statement is not more explicit than the one put forward in (a) above.

\section{The parity invariant}

The Moebius-Kantor graph is an incidence graph with many interesting features (several of which we exploited in [5] already) which are reflected in the properties of the corresponding Moebius-Kantor complexes. In the present section we define a metric (and simplicial) invariant for Moebius-Kantor complexes, called the parity (Def. 2.2), related to the relative disposition of roots in these complexes.

Let $X$ be a Moebius-Kantor complex. For convenience, we shall call 2-triangle a simplicial equilateral triangle in $X$ whose sides have length 2. Every 2-triangle contains 4 faces which are themselves (small) equilateral triangles.

Let $x \in X$ be a vertex and $L_{x}$ the link at $x$ (namely, the sphere of small radius around $x$ in $X$, endowed with the angular metric). Recall that we call root at 
$x$ a metric embedding $\alpha:[0, \pi] \hookrightarrow L_{x}$ in the link of $x$, such that $\alpha(0)$ is a link vertex. Every root has a $\operatorname{rank} \operatorname{rk}(\alpha)$ which is a rational number in $[1,2]$. It is defined by

$$
\operatorname{rk}(\alpha)=1+\frac{N(\alpha)}{q_{\alpha}}
$$

where

$$
N(\alpha):=\left|\left\{\beta \in \Phi_{x} \mid \alpha \neq \beta, \alpha(0)=\beta(0), \alpha(\pi)=\beta(\pi)\right\}\right|,
$$

writing $\Phi_{x}$ for the set of roots at $x$ and, for a root $\alpha, q_{\alpha}$ for the valency of $\alpha(0)$ minus 1:

$$
q_{\alpha}:=\operatorname{val}(\alpha(0))-1 .
$$

We refer to $[6, \S 4]$ for more details on these definitions. In a Moebius-Kantor complex, the rank of a root can be 2 or $\frac{3}{2}$. (This is straightforward to verify, and reflects the fact that in the Moebius-Kantor configuration, a non-incident point-line configuration determines either a single, or three triangles.) We say that these complexes have (local) rank $\frac{7}{4}$, which corresponds to the average rank of the roots in the root system $\Phi_{x}$.

Every side in a 2-triangle defines two roots at every vertex $x$ of its center (small) triangle, which are of the form $\alpha(t)$ and $\alpha(\pi-t)$ for $t \in[0, \pi]$ for some $\alpha \in \Phi_{x}$. These two roots have the same rank, which we call the rank of the corresponding side of the 2-triangle.

If $T_{1}, T_{2}$ are two 2-triangles, we say that $T_{1}$ and $T_{2}$ are in branching configuration if the intersection $T_{1} \cap T_{2}$ contains 3 triangles. In this case, we call branching permutation, and we write $T_{1} \rightarrow T_{2}$, the transformation that fixes the intersection $T_{1} \cap T_{2}$ pointwise, and takes the free triangle in $T_{1}$ isometrically to the free triangle in $T_{2}$. Such a transformation is involutive. The rank of the two sides of $T_{1}$, which are not included in the fixed set $T_{1} \cap T_{2}$, must change under such a transformation:

Lemma 2.1. Let $X$ be a Moebius-Kantor complex. Let $T_{1}, T_{2}$ be two 2-triangles of $X$ in branching configuration. If $\alpha$ is the root of a side of $T_{1}$ and $s: T_{1} \rightarrow T_{2}$ is a branching permutation, then the corresponding root transformation $\alpha \mapsto$ $s(\alpha)$ permutes the values of the rank:

$$
\left\{\begin{array}{l}
\frac{3}{2} \mapsto 2 \\
2 \mapsto \frac{3}{2}
\end{array}\right.
$$

unless $s(\alpha)=\alpha$.

Proof. Consider such a root $\alpha$ at a vertex $x$. In the link $L_{x}$, every isometric embedding $\alpha:\left[0, \frac{2 \pi}{3}\right] \hookrightarrow L_{x}$, where $\alpha(0)$ is a vertex, admits exactly two isometric extensions $\alpha:[0, \pi] \hookrightarrow L_{x}$ into a root. One of them has rank $\frac{3}{2}$, the other rank 2 . Hence the two values of the rank are permuted by a branching permutation. 
Using Lemma 2.1 one can define a metric invariant of the complex taking values in $\mathbb{Z} / 2 \mathbb{Z}$, and attached to every face of $X$.

Definition 2.2. The parity of a triangle face $t$ in a Moebius-Kantor complex is the parity of the number of roots of rank 2 in a 2 -triangle $T$ in which $t$ embeds as the centerpiece.

It follows from Lemma 2.1 that this is a well defined map

$$
X^{2} \rightarrow \mathbb{Z} / 2 \mathbb{Z}
$$

where $X^{2}$ denotes the set of 2 -faces of the 2-complex $X$. We call this map the parity map. It is clearly an invariant of isomorphism:

Lemma 2.3. Simplicial isomorphisms between Moebius-Kantor complexes preserve the parity of faces.

In particular, the automorphism group $\operatorname{Aut}(X)$ of a complex $X$ of rank $\frac{7}{4}$ acts in a parity preserving way.

Definition 2.4. We will say that a Moebius-Kantor complex is even (resp. odd) if its faces are even (resp. odd).

In general, one might expect (compare Sect. 5) that a Moebius-Kantor complex has mixed parity.

\section{Explicit computations}

In $[5, \S 4]$ we described thirteen constructions of Moebius-Kantor complexes which are transtive on vertices. The parity map can be computed explicitly in these examples, which provides new information on these complexes. We explain how in this section.

The simplest case to consider is that of $X_{0}:=\tilde{V}_{0}$. The complex can be described by its fundamental set of triangles as follows:

$$
V_{0}:=[[1,2,6],[2,3,7],[3,4,8],[4,5,1],[5,6,2],[6,7,3],[7,8,4],[8,1,5]] .
$$

It is the simplest case because the automorphism group is face transitive, and we know in advance that the complex $X_{0}$ is either odd or even. (In the definition of $V_{0}$, the triples represent oriented triangle faces with edges labelled with numbers, as indicated, and the complex $V_{0}$ is the quotient by the equivalence relation which identifies isometrically every triple of oriented edges with a given label; the space $V_{0}$ is a presentation complex and classifying space for $\pi_{1}\left(V_{0}\right)$.)

Proposition 3.1. $X_{0}$ is odd. 
Proof. Consider for instance the face $[1,2,6]$. It is contained in $8\left(=2^{3}\right) 2$ triangles. If the face $[1,2,6]$ is oriented counterclockwise, one of these 2triangles is given by the faces $[2,3,7],[6,7,3]$, and $[4,5,1]$ in this order. Labelling the link vertices by a signed integer $\pm k$, where $k \in\{1, \ldots, 8\}$, corresponding to an incoming $(+k)$ or a outgoing $(-k)$ edge with label $k$ in $X_{0}$, this gives us three roots at the vertices of $[1,2,6]$

$$
\begin{aligned}
& \alpha_{1}: 3 \rightarrow-6 \rightarrow 2 \rightarrow-3 \\
& \alpha_{2}: 5 \rightarrow-1 \rightarrow 6 \rightarrow-7 \\
& \alpha_{3}: 7 \rightarrow-2 \rightarrow 1 \rightarrow-4
\end{aligned}
$$

corresponding to the sides of the given 2-triangle. A direct computation in the labelled link using $(\dagger)$ shows that

$$
\begin{aligned}
& \operatorname{rk}\left(\alpha_{1}\right)=\operatorname{rk}\left(\alpha_{3}\right)=\frac{3}{2} \\
& \operatorname{rk}\left(\alpha_{2}\right)=2 .
\end{aligned}
$$

where the representation of the link and its labelling is given by

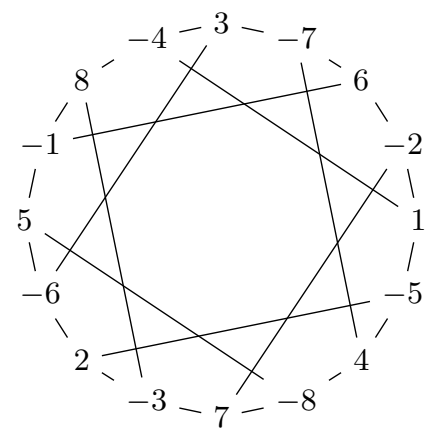

This proves that $X_{0}$ is odd.

This proof works for every face in any Moebius-Kantor complex. Similar computations, for example, will lead to the following statement.

Proposition 3.2. The following Moebius-Kantor complexes are even:

$$
\begin{aligned}
V_{0}^{1} & =[[1,2,3],[1,4,5],[1,6,4],[2,6,8],[2,8,5],[3,6,7],[3,7,5],[4,8,7]] \\
V_{0}^{2} & =[[1,2,3],[1,4,5],[1,6,7],[2,4,6],[2,8,5],[3,6,8],[3,7,5],[4,8,7]] \\
\check{V}_{0}^{2} & =[[1,2,3],[1,4,5],[1,6,7],[2,6,4],[2,8,5],[3,6,8],[3,7,5],[4,8,7]] \\
V_{4}^{1} & =[[1,1,5],[2,2,5],[3,3,6],[4,4,6],[1,3,8],[2,7,4],[5,8,7],[6,7,8]]
\end{aligned}
$$

The classification given in $\S 4$ of [5] contains eight other Moebius-Kantor complexes. These complexes are of mixed parity. The details of the parity maps are given in an appendix. 


\section{Constructing universal covers}

Our goal in this section is to use the parity to construct compact MoebiusKantor complexes with non isomorphic universal covers.

Theorem 4.1. There exists infinitely many compact Moebius-Kantor complexes with pairwise non isomorphic universal covers.

An idea is to construct complexes with "different proportions" of even faces, which will therefore have non isomorphic universal covers. A direct implementation of this requires to assign to a Moebius-Kantor complex, in a computable way, an average number of the even faces it contains. In view of the discussion in the introduction, we shall rather use the following invariant, which measures the 'radius of evenness' of the complex $X$.

Definition 4.2. Let $X$ be a simply connected Moebius-Kantor complex. We let $e(X)$ be the largest (possibly infinite) integer $n$ such that there exists a vertex $x \in X$ for which the ball $B(x, n)$ of radius $n$ is even.

Clearly, if $e(X) \neq e(Y)$, the complexes $X$ and $Y$ and not isomorphic. To prove the theorem, we will construct a sequence $X_{n}$ of universal covers on which $e$ is injective.

Lemma 4.3. Let $X$ be a simply connected Moebius-Kantor complex with cocompact isometry group. Then $X$ is even if and only if $e(X)=\infty$.

Proof. It is clear that $e(X)=\infty$ if $X$ is even. Conversely, assume that $e(X)=$ $\infty$. Then there exists a sequence $\left(x_{n}\right)$ of vertices of $X$ and a sequence $r_{n} \rightarrow \infty$ of radii, such that the ball $B\left(x_{n}, r_{n}\right)$ in $X$ are even. Since $\operatorname{Isom}(X)$ has a relatively compact fundamental domain, we may find a vertex $x$ in $X$ such that for every $n$, the balls $B\left(x, r_{n}\right)$ in $X$ are even. Thus, $X$ is even.

In order to find an injectivity set for $e$ we will use the surgery construction of [7]. In fact, this is the only construction of (infinitely many) compact MoebiusKantor complexes that we are aware of at this moment.

Let us start with a brief review of these constructions for groups acting on Moebius-Kantor complexes. The surgery is described by a category Bord $_{\frac{7}{4}}$ whose arrows are called group cobordisms, and whose objects are called collars. Both the objects and the morphisms in $\operatorname{Bord}_{\frac{7}{4}}$ correspond to 2-dimensional complexes.

The following 2-complex defines a collar in Bord $_{\frac{7}{4}}$. It was used in [8] to define a model of random groups acting on Moebius-Kantor complexes:

$$
(x, a, d),(y, c, d),(z, c, b),\left(x^{\prime}, d, a\right),\left(y^{\prime}, b, a\right),\left(z^{\prime}, b, c\right)
$$

This 2-complex $C$ is obtained from a set of six oriented equilateral triangles, with labeled edges, by identifying the edges respecting the labels and the orientations. 
This collar was identified in [7], where it was realized (see Lemma 11.1 in this paper) as the separating collar of the following Moebius-Kantor complex

$$
\begin{aligned}
& {[[1,11,3],[2,12,4],[1,15,12],[3,6,4],[3,7,6],[4,6,8],[5,7,8],[5,8,7],} \\
& {[11,1,13],[12,2,14],[11,5,2],[13,16,14],[13,17,16],} \\
& [14,16,18],[15,17,18],[15,18,17]] .
\end{aligned}
$$

As explained in [7], the latter complex is a 2-sheeted cover of one of the thirteen complexes found in [5]. This shows, in particular, that $C$ is an object of $\operatorname{Bord}_{\frac{7}{4}}$.

We will use two arrows

$$
X_{00}, Y_{00}: C \rightarrow C
$$

in $\operatorname{Bord}_{\frac{7}{4}}$. We maintain the notation of $[8, \S 2]$ for consistency. As 2-complexes, these arrows have the following respective presentations

$$
\begin{aligned}
& (x, a, d),(y, c, d),(z, c, b),(1,1,2),\left(2, a^{\prime}, d^{\prime}\right),\left(4, c^{\prime}, d^{\prime}\right),\left(3, c^{\prime}, b^{\prime}\right) \\
& (4, d, a),(3, b, a),(2, b, c),(1,3,4),\left(x^{\prime}, d^{\prime}, a^{\prime}\right),\left(y^{\prime}, b^{\prime}, a^{\prime}\right),\left(z^{\prime}, b^{\prime}, c^{\prime}\right)
\end{aligned}
$$

and

$$
\begin{aligned}
& (x, a, d),(y, c, d),(z, c, b),(1,2,3), \quad\left(4, a^{\prime}, d^{\prime}\right),\left(2, c^{\prime}, d^{\prime}\right),\left(1, c^{\prime}, b^{\prime}\right) \\
& (1, d, a),(3, b, a),(4, b, c),(2,4,3),\left(x^{\prime}, d^{\prime}, a^{\prime}\right),\left(y^{\prime}, b^{\prime}, a^{\prime}\right),\left(z^{\prime}, b^{\prime}, c^{\prime}\right)
\end{aligned}
$$

described in [8, Remark 2.8]. Again, these are 2-complexes obtained by identifying the edges of the given sets of equilateral triangles, respecting the labels and the orientations. As group cobordisms, i.e., when viewed as arrows $C \rightarrow C$ in the category $\operatorname{Bord}_{\frac{7}{4}}$ (see $[7, \S 10]$ ), these complexes are augmented with maps $L: C \rightarrow Z$ and $R: C \rightarrow Z$, which in the case of $Z=X_{00}$ and $Z=Y_{00}$ are the obvious ones.

As in Sect. 3, Propostion 3.1, a direct computation shows that:

Lemma 4.4. The triangles $(1,1,2)$ and $(1,3,4)$ (resp. $(1,2,3)$ and $(2,3,4)$ ) are even in $X_{00}$ (resp. in $\left.Y_{00}\right)$.

Note that the cobordisms $X_{00}$ and $Y_{00}$ themselves are not Moebius-Kantor complexes, since they have a non trivial boundary. However, since the triangles referred to in the above lemma belong to the core, the link considered is indeed the Moebius-Kantor graph, and the parity makes sense in this case.

On the other hand, the parity of the collar faces is not determined a priori by the cobordism alone, since it may depend on composition. We shall use this fact to construct complexes with different parity ratios.

Lemma 4.5. In the composition $Y_{00} Y_{00}$, the central collar is even.

Proof. Indeed, the $n$-fold composition $\underbrace{Y_{00} \cdots Y_{00}}_{n}$ of the cobordism $Y_{00}$ leads to finite covers of the $V_{0}^{1}$, which is an even complex, as we noted in Propostion 3.2. 
On the other hand we have:

Lemma 4.6. The central collar in the composition $X_{00} Y_{00}$ contains an odd face.

Proof. Let us show that the face $\left(4, c^{\prime}, d^{\prime}\right)$ is odd. We can relabel the second cobordism $Y_{00}$ as follows

$$
\begin{aligned}
& \left(2, a^{\prime}, d^{\prime}\right),\left(4, c^{\prime}, d^{\prime}\right),\left(3, c^{\prime}, b^{\prime}\right),(11,12,13),\left(14, a^{\prime \prime}, d^{\prime \prime}\right),\left(12, c^{\prime \prime}, d^{\prime \prime}\right),\left(11, c^{\prime \prime}, b^{\prime \prime}\right) \\
& \left(11, d^{\prime}, a^{\prime}\right),\left(13, b^{\prime}, a^{\prime}\right),\left(14, b^{\prime}, c^{\prime}\right), \\
& (12,14,13),\left(x^{\prime \prime}, d^{\prime \prime}, a^{\prime \prime}\right),\left(y^{\prime \prime}, b^{\prime \prime}, a^{\prime \prime}\right),\left(z^{\prime \prime}, b^{\prime \prime}, c^{\prime \prime}\right)
\end{aligned}
$$

viewing it as embedded in the composition $X_{00} Y_{00}$. A 2-triangle containing this face is described, in counterclockwise order, by the 3 faces $(1,3,4),\left(3, c^{\prime}, b^{\prime}\right)$, and $\left(2, a^{\prime}, d^{\prime}\right)$, which gives us three roots

$$
\begin{aligned}
& \alpha_{1}: a^{\prime} \rightarrow-d^{\prime} \rightarrow c^{\prime} \rightarrow-b^{\prime} \\
& \alpha_{2}: 3 \rightarrow-c^{\prime} \rightarrow 4 \rightarrow-1 \\
& \alpha_{3}: 3 \rightarrow-4 \rightarrow d^{\prime} \rightarrow-2
\end{aligned}
$$

of which we have to compute the rank.

The root $\alpha_{1}$ belongs to the following link in $X_{00} Y_{00}$ :

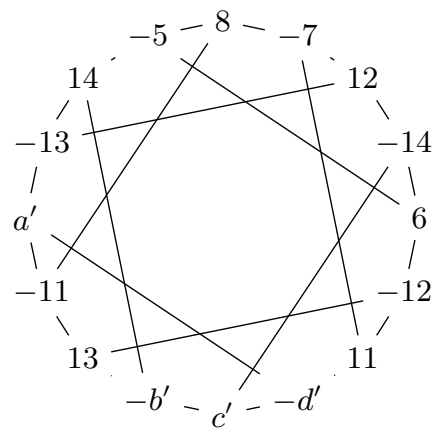

Therefore it is of rank 2 .

The roots $\alpha_{2}$ and $\alpha_{3}$ belong to the following link:

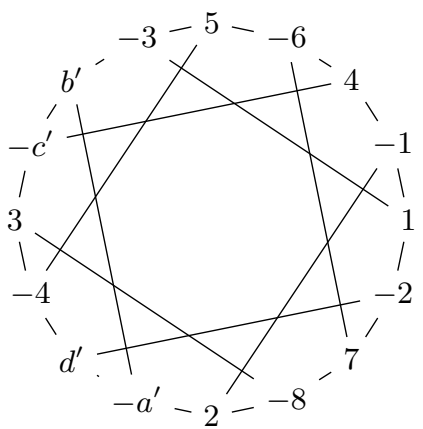


Both of them are of rank $\frac{3}{2}$.

This proves that the face is odd.

Proof of Theorem 4.1. Consider the compact complexe of rank $\frac{7}{4}$

$$
V_{n}:=X_{00} \underbrace{Y_{00} \cdots Y_{00}}_{2 n} / \sim
$$

where $\sim$ identifies the two extremal copies of $C$ in the composition $X_{00} Y_{00} \cdots Y_{00}$. Let $X_{n}$ denote the universal cover of $V_{n}$. Since the projection $X_{n} \rightarrow V_{n}$ reduces the distances, it is clear that if $x_{n}$ denotes a lift the core vertex in the $n^{\text {th }}$ cobor$\operatorname{dism} Y_{00}$ in $V_{n}$, then by Lemma 4.5 the ball of radius $n$ in $X_{n}$ is even. It follows that $e\left(X_{n}\right) \geq n$. By Lemmas 4.3 and 4.6 , we have $e\left(X_{n}\right)<\infty$. Therefore, we can find a subsequence of $\left(V_{n}\right)_{n}$ on which $e$ is injective.

Remark 4.7. (1) By their definition, the groups $\pi_{1}\left(V_{n}\right)$ in Theorem 4.1 are accessible by surgery in the sense of [7].

(2) The proof shows that the universal cover of $V_{0}^{1}$ is not determined locally among the Moebius-Kantor complexes, namely one can find compact complexes of rank $\frac{7}{4}$ with universal cover distinct from $X_{0}^{1}$, but which coincide with it on arbitrary large balls. Another way to phrase this is to say that $V_{0}^{1}$ is an accumulation point in the space of Moebius-Kantor complexes.

(3) Lemma 4.5 shows that the universal covers constructed in the present section always contain a definite amount of even faces, which can never be reduced to zero. On the other hand, we have an example $X_{0}=\tilde{V}_{0}$ of a complex with no even face (Propostion 3.1). In the forthcoming section we show how to construct (simply connected) Moebius-Kantor complexes with an arbitrary parity map.

\section{Free constructions}

J. Tits made the observation that certain free constructions of (e.g.) Euclidean buildings could be given, in analogy with the construction of free projective planes and generalized polygons, in particular for buildings lacking irreducible spherical residues of type $A_{3}, C_{3}$ or $H_{3}$ (Tits did not published these resultscompare $[12,13]$ for an announcement).

In fact, it was proved by Ronan [9] that under these assumptions (on the rank 3 residues), all buildings can be obtained by a universal free construction, and furthermore, that a prescription theorem holds, to the effect that the residues at the vertices can be taken to run over any set of projective plane of a given order. These results showed, in particular, that there is complete freedom in the constructions of buildings of type $\tilde{A}_{2}$. 
We refer to $[9,10]$ for more information on these theorems, to the work of van Maldeghem [15] for a characterization using valuations on planar ternary rings in the $\tilde{A}_{2}$ case, and to $[2, \S 2]$ for a different free prescription theorem along these lines, for buildings of type $\tilde{A}_{2}$ and order 2 .

Our goal in this section is to show that the Moebius-Kantor complexes behave similarly in this respect. We prove a similar prescription theorem, replacing preassigned sets of projective planes with preassigned parity maps; the result can be stated, informally, as follows.

Theorem 5.1. There exist "free constructions" of simply connected MoebiusKantor complexes with prescribed parity map; in particular, there exist uncountably many pairwise non isomorphic simply connected Moebius-Kantor complexes.

These free constructions are (as in the above references) by induction, starting with a ball $B_{1}$ in a Moebius-Kantor complex and extending successively the balls $B_{1} \subset B_{2} \subset \cdots$, and setting $X:=\bigcup B_{n}$, where we have to show that the parity can be chosen freely. This is done by means of the following lemma, from which the theorem follows easily.

Lemma 5.2. Let $B_{n}$ be a ball of radius $n$ centered at a vertex in a MoebiusKantor complex, and let $S_{n}:=B_{n} \backslash\left(B_{n-1}\right)^{\circ}$ denote the closed simplicial sphere of radius $n$, where $\left(B_{n-1}\right)^{\circ}$ denotes the interior of $B_{n-1}$. Let $p: S_{n} \rightarrow \mathbb{Z} / 2 \mathbb{Z}$ be a map defined on the 2-skeleton. Then there exists a ball $B_{n+1}$ in a MoebiusKantor complex, containing $B_{n}$, and such that the parity of the faces of $S_{n}$ in $B_{n+1}$ is given by $p$.

Thus, the "free constructions" referred to in Theorem 5.1 are defined in the obvious way, starting with $B_{1}$ and a fixed prescription $p_{1}$ of the parity, using the lemma to extend $B_{1} \subset B_{2}$ to realize $p_{1}$, prescribe a new parity map $p_{2}$ arbitrarily on $S_{2}$, and iterate ad infinitum this process, applying the lemma infinitely many times, to define, simultaneously, the complex $X:=\bigcup B_{n}$ together with a freely prescribed direct limit parity map $p:=\lim _{\longrightarrow} p_{1}+\cdots+p_{n}$.

Proof. If $f$ is a face in $S_{n}$, then $f \cap \partial B_{n}$ is either a vertex or an edge (where $\partial B_{n}$ denotes the topological boundary). Suppose that $f \cap \partial B_{n}$ is a vertex $x$. Then $f$ is adjacent to 4 faces in $S_{n}$ whose intersections with $\partial B_{n}$ is an edge. Let $T$ be a 2 -triangle containing the face $f$ and let $\alpha$ be a root corresponding to $T$ at $x$. We can choose to embed $\alpha$ in the Moebius-Kantor graph $L_{x}$ in such a way that its rank is $\frac{3}{2}$ or 2 , and therefore can freely decide the parity of $f$ in the construction, which can be chosen to be $p(f)$. Furthermore, this operation only determines the following subgraph of $L_{x}$. 


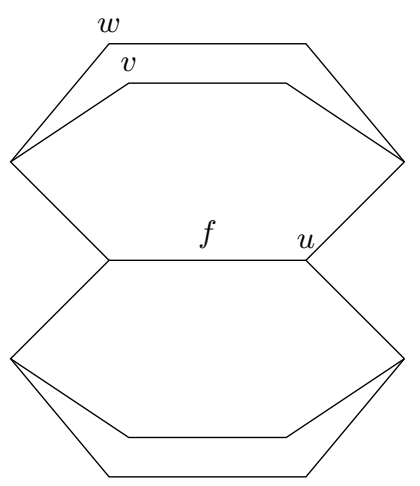

In this graph, the edge labelled $f$ corresponds to the centerpiece of the root $\alpha$, which extends on both side, in a direction which depends on its rank. The origin and extremity of $\alpha$ belong to the two faces in $T$ adjacent to $f$ and intersecting the boundary. Let $g$ be one of these two faces and $\beta$ be a root in $L_{x}$ corresponding to $g$. Note that $g$ is such that $g \cap \partial B_{n}$ is an edge. We have to show that the parity of $g$ can be chosen freely (according to $p$ ). By symmetry, one may assume that $\beta(0)=u$ and $\beta(\pi)=v$ or $w$. Lemma 2.1 ensures that the rank of $\beta$ can be permuted freely according to the choice made $\beta(\pi)=v$ or $\beta(\pi)=w$ under an embedding into the Moebius-Kantor graph. Thus, one is free to choose the rank parity of $g$, by choosing an appropriate embedding of the above graph in the MoebiusKantor graph $L_{x}$. We can then repeat this argument for the three other faces attached to $f$. The rank of the corresponding roots can be prescribed via an appropriate embedding of the graph shown above in the Moebius-Kantor graph.

In fact, this argument shows that for any face $g$ such that $g \cap \partial B_{n}$ is a single edge $[x, y]$, the rank of the two roots $\beta_{x}$ and $\beta_{y}$, corresponding to $x$ and $y$ in a 2-triangle containing $g$ in its center, can be chosen independently by appropriate choice of an embedding of $\beta_{x}$ and $\beta_{y}$ in the Moebius-Kantor graph. In particular, the parity of $g$ can be chosen to be $p(g)$.

Remark 5.3. (1) It seems clear from the proof that the parity alone will not determine the isomorphism class of a Moebius-Kantor complex, and that there ought to exist uncountably many complexes with given parity (for example, uncountably many even/odd complexes of rank 7/4). A formal proof of this assertion requires the use of a more refined invariant that distinguishes between complexes with a given parity map. The main advantage of using the parity is the fact that its values can be altered by changing the rank of either one of the roots $\beta_{x}$ or $\beta_{y}$ independently, which leads to total freedom of construction. For a more constrained invariant (such as the isomorphism type of the 2-balls), one may want to only prove partial prescription results (e.g., prescriptions at vertices not adjacent to 
a face $f$ such that $f \cap \partial B_{n}$ is a vertex assuming $n$ large enough). We shall not pursue these investigations further on this occasion.

(2) It follows from Theorem 5.1 that the equivalence in Lemma 4.3 fails without the assumption that the isometry group has compact quotient.

The following should be compared to the results of [14].

Corollary 5.4. There exists at least 174,163 isomorphism types of spheres of radius 2 in Moebius-Kantor complexes.

Proof. A sphere of radius 2 determines the parity map on the ball $B$ of radius 1. By Theorem 5.1, for every map $p: B \rightarrow \mathbb{Z} / 2 \mathbb{Z}$ defined on the 2 -skeleton, there exists a ball $B_{p}$ of radius 2 extending $B$, such that the parity of the faces of the ball of radius 1 in $B_{p}$ is given by $p$. Since the parity is an invariant of isomorphism, if $B_{p}$ and $B_{p^{\prime}}$ are abstractly isomorphic, then there exists an automorphism $\theta: B \rightarrow B$ such that $p^{\prime}=p \circ \theta$. Thus, the number of spheres of radius 2 is at least the number of $p$ 's modulo the action of $\operatorname{Aut}(B) \simeq \operatorname{Aut}(G)$ where $G$ is the Moebius-Kantor graph. This gives at least $2^{24} / 96=174,762.6$ isomorphism types.

\section{Generic constructions}

In $[3,4]$ we studied Euclidean buildings of type $\tilde{A}_{2}$ from a dynamical point of view, motivated by some questions in orbit equivalence theory. In view of the result in Sect. 5, it seems obvious that these results will carry over to Moebius-Kantor complexes. In the present section we mention an analog of [3, Theorem 5], which can be stated as follows:

Theorem 6.1. A generic Moebius-Kantor complex has trivial automorphism group.

The notion of genericity used in this result (and in [3]) is topological, in the space of Baire, meaning that this holds for a dense $G_{\delta}$ set in the space of pointed Moebius-Kantor complexes.

Rather than giving a formal proof of this result (which would significantly overlap with [3]), let us reproduce the figure from $[3, \S 6]$ since it is helpful to understand how the generic triviality of the automorphism group occurs. 


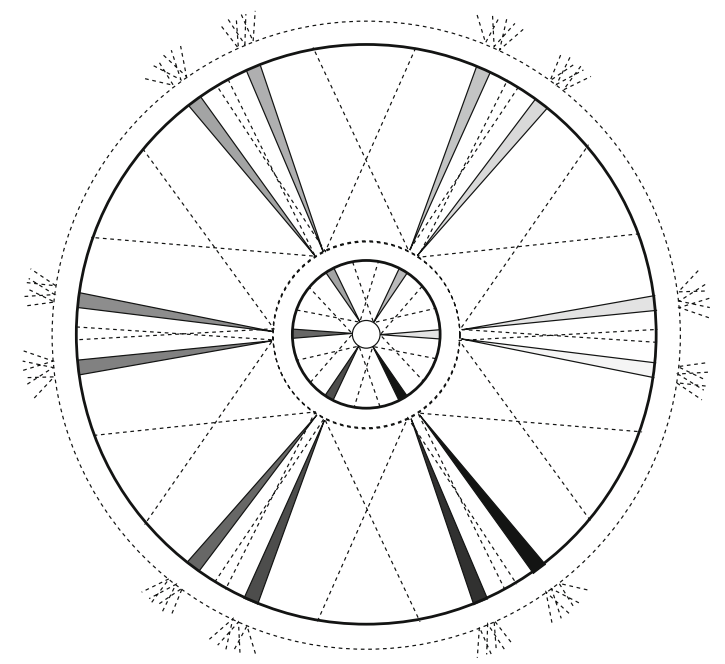

This figure symbolizes a Moebius-Kantor complex (or a building in the situation of [3]). The central (white) disk represents a ball of "small" (i.e., arbitrary large, but fixed) radius, which corresponds to fixing a neighborhood in the space of pointed Moebius-Kantor complexes. Above this ball are larger balls which we can control to trivialize automorphisms, where the shades of grey account for the "density" of odd faces in the given portion of the complex. With some details, that need to be checked (as we did in [3], in the case of buildings), this proves that the automorphism group of the generic (in the topological sense) space of rank $\frac{7}{4}$ is trivial.

Remark 6.2. (1) This argument is quite robust. It shows that the automorphism group of the spaces is generically trivial as soon as one can establish a free prescription theorem in the style of Ronan [9] (or at least a partial prescription theorem which offers enough, if not total freedom, as in [3] in the case $q \geq 3$ ), of a local invariant of isomorphism. The parity is such a local invariant of isomorphism, by Theorem 5.1.

(2) In the case of Moebius-Kantor complexes, it is easy to check that the isotropy groups are finite, and therefore the full use of shadings is not required. Namely, one may trivialize the isotropy groups by using appropriate shades of grey in the first wreath, and then confine oneself to prescribe even wreaths, followed by odd ones in alternance.

(3) The parity allows us to distinguish universal covers up to isometry, but due to its local nature, there seems to be no direct way to make use of it for quasi-isometries. It is of course natural to wonder what the quasiisometry types of Moebius-Kantor complexes can be.

(4) One motivation for studying the space of Euclidean buildings was, in the theory of orbit equivalence, to give new constructions of probability measure preserving standard equivalence relations with the property $\mathrm{T}$ of Kazhdan. One difficulty is the construction of diffuse invariant measures on this space. In [7] it is shown that the (similarly defined) space of 
Moebius-Kantor complexes, contrary to what we currently know in the case of Euclidean buildings, admits diffuse invariant measures, albeit ones with amenable support. It would be interesting to study quasiperiodic Moebius-Kantor spaces in more details, using the techniques of $[3,4]$ for example.

\section{Acknowledgements}

The authors are grateful to the referees for helpful suggestions and corrections. The second author was partially supported by an NSERC discovery Grant No. 418144-12 and a JSPS award.

Publisher's Note Springer Nature remains neutral with regard to jurisdictional claims in published maps and institutional affiliations.

\section{Appendix}

In this appendix we compute the parity map for the remaining eight MoebiusKantor complexes from [5], namely $V_{1}, V_{3}, V_{5}, V_{2}^{i}$ for $i=1, \ldots, 4$, and $V_{4}^{2}$.

\begin{tabular}{lllllllll}
\hline$V_{1}$ & {$[1,1,2]$} & {$[1,3,4]$} & {$[2,5,6]$} & {$[2,7,8]$} & {$[3,5,7]$} & {$[3,6,5]$} & {$[4,6,8]$} & {$[4,8,7]$} \\
\hline Parity & 0 & 0 & 1 & 1 & 0 & 0 & 0 & 0 \\
\hline
\end{tabular}

\begin{tabular}{lllllllll}
\hline$V_{2}^{1}$ & {$[1,1,3]$} & {$[2,2,3]$} & {$[1,4,5]$} & {$[2,7,8]$} & {$[3,5,7]$} & {$[4,6,8]$} & {$[4,7,6]$} & {$[5,8,6]$} \\
\hline Parity & 0 & 0 & 1 & 1 & 0 & 0 & 0 & 0 \\
\hline
\end{tabular}

\begin{tabular}{lllllllll}
\hline$V_{2}^{2}$ & {$[1,1,3]$} & {$[2,2,4]$} & {$[3,7,4]$} & {$[1,4,6]$} & {$[2,5,3]$} & {$[5,7,8]$} & {$[5,8,6]$} & {$[6,8,7]$} \\
\hline Parity & 1 & 1 & 0 & 1 & 1 & 0 & 0 & 0 \\
\hline
\end{tabular}

\begin{tabular}{lllllllll}
\hline$V_{2}^{3}$ & {$[1,1,3]$} & {$[2,2,4]$} & {$[1,5,2]$} & {$[3,6,4]$} & {$[3,7,6]$} & {$[4,6,8]$} & {$[5,7,8]$} & {$[5,8,7]$} \\
\hline Parity & 0 & 0 & 0 & 1 & 1 & 1 & 0 & 0 \\
\hline
\end{tabular}

\begin{tabular}{lllllllll}
\hline$V_{2}^{4}$ & {$[1,1,3]$} & {$[2,2,4]$} & {$[1,5,2]$} & {$[3,6,5]$} & {$[3,7,8]$} & {$[4,5,8]$} & {$[4,6,7]$} & {$[6,8,7]$} \\
\hline Parity & 0 & 0 & 0 & 1 & 1 & 1 & 1 & 0 \\
\hline
\end{tabular}




\begin{tabular}{lllllllll}
\hline$V_{3}$ & {$[1,1,4]$} & {$[2,2,4]$} & {$[3,3,5]$} & {$[1,3,6]$} & {$[2,5,7]$} & {$[4,7,8]$} & {$[5,8,6]$} & {$[6,8,7]$} \\
\hline Parity & 0 & 0 & 0 & 0 & 1 & 1 & 1 & 0 \\
\hline
\end{tabular}

\begin{tabular}{lllllllll}
\hline$V_{4}^{2}$ & {$[1,1,5]$} & {$[2,2,5]$} & {$[3,3,6]$} & {$[4,4,7]$} & {$[1,3,8]$} & {$[2,7,6]$} & {$[4,8,6]$} & {$[5,8,7]$} \\
\hline Parity & 0 & 0 & 1 & 1 & 0 & 1 & 1 & 1 \\
\hline
\end{tabular}

\begin{tabular}{lllllllll}
\hline$V_{5}$ & {$[1,1,2]$} & {$[3,3,2]$} & {$[4,4,2]$} & {$[5,5,6]$} & {$[7,7,8]$} & {$[1,8,6]$} & {$[3,6,7]$} & {$[5,8,4]$} \\
\hline Parity & 0 & 0 & 0 & 1 & 1 & 0 & 0 & 0 \\
\hline
\end{tabular}

Remark 7.1. The complex $V_{0}$ is therefore the unique complex in the list of [5] such that every face is the centerpiece of a 2-triangle whose three sides are of rank 2 .

\section{References}

[1] Barré, S.: Polyèdres de rang deux. Thèse, ENS Lyon, Lyon (1996)

[2] Barré, S.: Immeubles de tits triangulaires exotiques. In: Annales de la Faculté des sciences de Toulouse: Mathématiques, vol. 9, pp. 575-603. Université Paul Sabatier, Toulouse (2000)

[3] Barré, S., Pichot, M.: Sur les immeubles triangulaires et leurs automorphismes. Geom. Dedicata. 130(1), 71-91 (2007)

[4] Barré, S., Pichot, M.: Existence d'immeubles triangulaires quasi-périodiques. Math. Ann. 350(1), 227-242 (2011)

[5] Barré, S., Pichot, M.: Intermediate rank and property RD. arXiv:0710.1514

[6] Barré, S., Pichot, M.: Removing chambers in bruhat-tits buildings. Israel J. Math. 202, 117-160 (2014)

[7] Barré, S., Pichot, M.: Surgery on discrete groups, preprint.

[8] Barré, S., Pichot, M.: Random group cobordisms of rank $\frac{7}{4}$, preprint.

[9] Ronan, M.A.: A construction of buildings with no rank 3 residues of spherical type. In: Buildings and the Geometry of Diagrams, pp. 242-248. Springer, Berlin (1986)

[10] Ronan, M.A., Tits, J.: Building buildings. Math. Ann. 278(1-4), 291-306 (1987)

[11] Tits, J.: Buildings and $B N$-pairs of spherical type. Lecture Notes in Mathematics 386, (1974)

[12] Tits, J.: Endliche spiegelungsgruppen, die als weylgruppen auftreten. Invent. Math. 43(3), 283-295 (1977)

[13] Tits, J.: A local approach to buildings. In: The Geometric Vein, pp. 519-547. Springer, Berlin (1981)

[14] Tits, J.: Spheres of radius 2 in triangle buildings. In: Finite Geometries Buildings and Related Topics. Pingrie Park Conference (1988) 
[15] Van Maldeghem, H.: Non-classical triangle buildings. Geom. Dedicata. 24(2), 123-206 (1987)

Sylvain Barré

UMR 6205, LMBA, UniversitÉ de Bretagne-Sud

BP 573

56017 Vannes

France

e-mail: Sylvain.Barre@univ-ubs.fr

Mikaël Pichot

McGill University

805 Sherbrooke St W.

Montréal

QC H3A 0B9

Canada

e-mail: mikael.pichot@mcgill.ca 